Acknowledgments. We would like to thank Editage (www.editage.com) for English language editing. The views expressed in this letter are our own personal opinion, written in our private capacity, and do not necessarily reflect the views of my employer.

Financial support. No financial support was provided relevant to this article.

Conflicts of interest. The authors work for LSI Medience, a clinical laboratory in Tokyo, Japan that conducts clinical tests including PCR tests for COVID-19.

\section{References}

1. Coronavirus disease (COVID-19) Pandemic World Health Organization website. https://www.who.int/emergencies/diseases/novel-coronavirus-2019. Accessed April 6, 2020.
2. Tanabe K. Society coexisting with COVID-19. Infect Control Hosp Epidemiol. 2020 Apr 13 [Epub ahead of print]. doi: 10.1017/ice.2020.130.

3. Miller A, Reandelar MJ, Fasciglione K, Roumenova V, Li Y, Otazu GH. Correlation between universal BCG vaccination policy and reduced morbidity and mortality for COVID-19: an epidemiological study. MedRxiv 2020 Apr 6 [Preprint]. doi: 10.1101/2020.03.24.20042937.

4. Stadler K, Masignani V, Eickmann M, et al. SARS—beginning to understand a new virus. Nat Rev Microbiol 2003;1:209-18.

5. Zumla A, Hui DS, Perlman S. Middle East respiratory syndrome. Lancet 2015;386:995-1007.

6. Park HY, Lee EJ, Ryu YW, et al. Epidemiological investigation of MERS-CoV spread in a single hospital in South Korea, May to June 2015. Euro Surveill 2015;20:1-6.

\title{
A case of COVID-19 with an ultralong incubation period
}

\author{
Yujin Wang $\mathrm{MS}^{1,2, a}$, Qingwen Wang $\mathrm{MS}^{1,2, a}$, Kai Wang $\mathrm{MS}^{3}$, Congkuan Song $\mathrm{MS}^{1,2}$, ZiXin Guo $\mathrm{MS}^{1,2}$ and \\ Weidong Hu PhD, MD ${ }^{1,2}$ (D) \\ ${ }^{1}$ Department of Thoracic Surgery, ZhongNan Hospital of Wuhan University, Wuhan, Hubei, China, ${ }^{2}$ Hubei Key Laboratory of Tumor Biological Behaviors, Hubei \\ Cancer Clinical Study Center, Hubei, China and ${ }^{3}$ Township government of Shanpo, Jiangxia District, Wuhan, Hubei, China
}

To the Editor-A large global outbreak of coronavirus disease 2019 (COVID-19), caused by SARS-CoV-2, has become a critical public health issue since December 2019., ${ }^{1,2}$ SARS-CoV-2 spreads by human-to-human transmission mainly via droplets or direct contact, and it has been estimated to have mean incubation period of 6.4 days and a basic reproduction number of 2.24-3.58. ${ }^{3,4}$ An understanding of the incubation period is essential to detect epidemiological cases and helpful to determine the quarantine and medical observation period of intimate contacts. Whether SARS-CoV-2 can be transmitted during the incubation period remains controversial. ${ }^{5-7}$ Here, we report the case of a patient who had a long incubation period (38 days) and infected 1 close contact with SARS-CoV-2 during the incubation period.

A 50-year-old man (person 1), who worked in Hankou District, Wuhan, China, returned home on January 21, 2020, to a small village in the rural area of Wuhan City. First, he took the city subway, then he boarded an intercity train to a rural town, and finally his brother-in-law (person 2) drove him home by car. After returning home, person 1 lived with his elder brother (person 3), who was a single man with mild deafness who lived alone during ordinary (nonoutbreak) times. From then on, both men did not leave their home. Person 3 lived in an independent house in the small village and had neither gone to other places nor had contacted other people since January 1, 2020. Person 2 sent some food to the house of person 3 on January 17, but at that time, person 3 was not at home because he was doing farm work in the field. Persons 1 and 3 were at home together beginning January 21, 2020, and during this period they met nobody.

Author for correspondence: Weidong Hu MD, PHD, E-mail address: huwd@whu.edu.cn ${ }^{\text {a}}$ Authors of equal contribution.

Cite this article: Wang Y, et al. (2021). A case of COVID-19 with an ultralong incubation period. Infection Control \& Hospital Epidemiology, 42: 242-243, https:// doi.org/10.1017/ice.2020.221
Person 3 developed symptoms of cough and wheezing on February 5, 2020. On February 6, he underwent a chest computed tomography (CT) examination and was suspected of COVID-19 pneumonia. Person 3 was admitted to the hospital for treatment on February 7, and the next day his throat swab test for SARS-CoV-2 was positive by quantitative RT-PCR (qRT-PCR) analysis. During the hospitalization, person 3 was in stable condition without severe complications such as acute respiratory distress syndrome or shock. After treatment, person 3 was discharged on February 23 and isolated himself.

Person 1 began a 14-day quarantine on February 7 and ended on February 21. On February 24, he underwent a routine CT examination before returning home. The results showed very slight inflammation of the lung, but at that time, he still did not have any symptoms. On February 27, person 1 started to have a cough, and he underwent another chest CT test, which indicated progressive pulmonary inflammation, so he was admitted to the hospital for quarantine and treatment. His SARS-CoV-2 test was also positive by qRT-PCR analysis. After treatment, he had 2 throat swab test for SARS-CoV-2 a week apart. The results of these tests were negative, and another chest CT image showed that the inflammation was absorbing. Thus, person 1 was discharged on March 2 .

Person 2 was the other man who had initial contact with person 1 on January 21 . He lived with his family (his wife and son) from then on, and he had a family dinner with his other 2 relatives (his brother and sister-in-law) on January 24. These 5 people were in good health until March 6. Under strict isolation control, the villagers basically had no contact, and no other people were reported to have COVID-19 in the village where person 1 and person 3 lived (Fig. 1).

Except for person 1, who came from the Hankou District of Wuhan, the epidemic area on January 21, when COVID-19 was rapidly spreading, person 3 had no contact with other people, especially with infected or suspected persons, for more than a month 


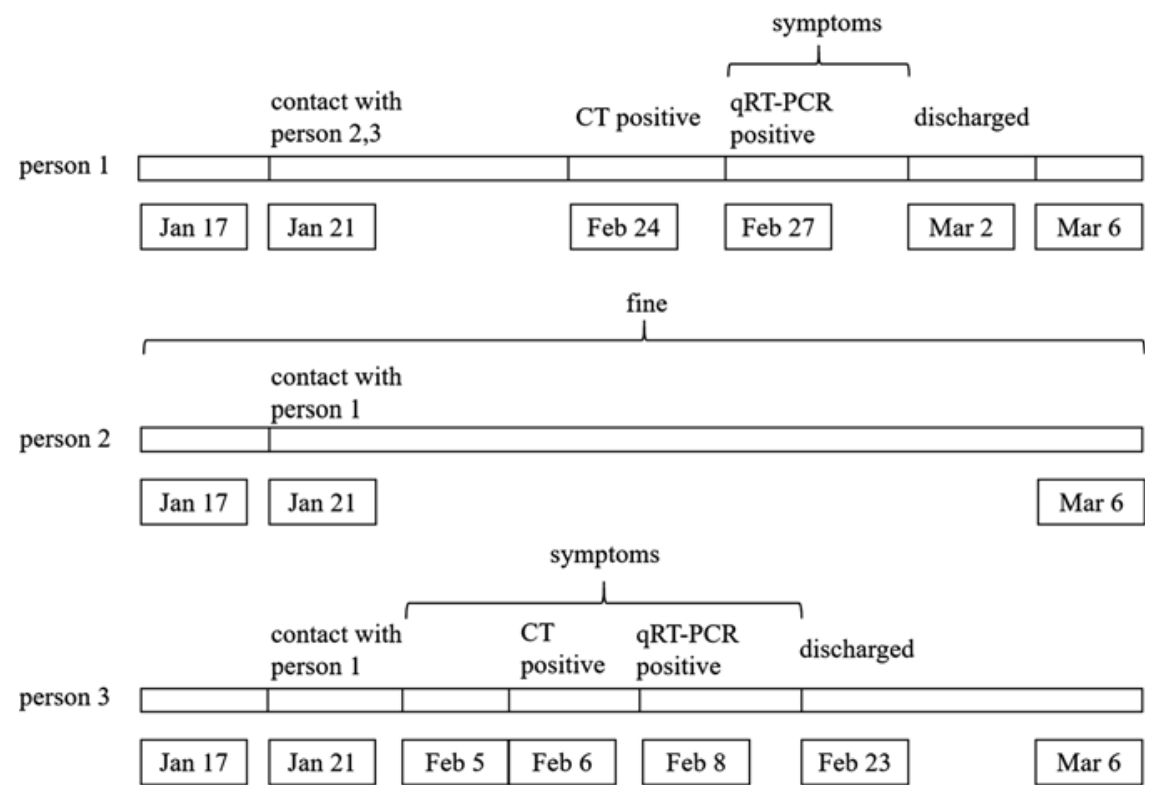

Fig. 1. Chronology of contacts, symptom onset and identification of positive SARS-CoV-2 findings on CT and qRT-PCR of 3 people. Note. CT, computed tomography; qRT-PCR, quantitative reverse-transcriptase polymerase chain reaction. before he was identified of COVID-19 on February 8. Person 3's infection was suspected to have come from person 1, of whom the incubation period was 16 days.

Based on the exposure history and the onset of symptoms of person 1 , we reasonably speculated that the incubation period of person 1 was at least 38 days, which was calculated from January 21 to February 27. By contrast, the incubation period of these 2 patients was much longer than the mean incubation period reported by Backer et $\mathrm{al}^{3}$ and person 1's case may represent the longest incubation period of all cases reported up to now. ${ }^{8,9}$

The long incubation period and mild symptoms of these 2 patients is notable and may be due to the weak virulence of the SARS-CoV-2 type to which they were exposed. Tang et al ${ }^{10}$ divided SARS-CoV-2 into 2 major types based on population genetic analyses of 103 SARS-CoV-2 genomes, 1 of which was less aggressive. The weak virulence of the virus is a double-edged sword. On one hand, it poses less threat to the infected, and on the other hand, people may carry the virus for a long time without realizing it and therefore transmit it to others inadvertently.

In this report, person 3 was infected by person 1 during the incubation period, which provides evidence that SARS-CoV-2 was contagious during the incubation period. The long incubation period of this COVID-19 case may contribute to our knowledge of SARS-CoV-2 and may imply that a longer observation or isolation period for contacts should be considered in SARS-CoV-2 epidemic areas.

\section{Acknowledgments. None.}

Financial support. This study was supported by Science Technology and Innovation Cultivating Fund of Zhongnan Hospital of Wuhan University (grant no. cxpy2017041).
Conflicts of interest. The authors declare no conflict of interest related to this letter.

\section{References}

1. Hui DS, Azhar EI, Madani TA, et al. The continuing 2019-nCoV epidemic threat of novel coronaviruses to global health-the latest 2019 novel coronavirus outbreak in Wuhan, China. Int J Infect Dis 2020;91:264-266.

2. Rothan HA, Byrareddy SN. The epidemiology and pathogenesis of coronavirus disease (COVID-19) outbreak. J Autoimmun 2020;109:102433.

3. Backer JA, Klinkenberg D, Wallinga J. Incubation period of 2019 novel coronavirus (2019-nCoV) infections among travellers from Wuhan, China. Euro Surveill 2020;25(5):2000062.

4. Zhao S, Lin Q, Ran J, et al. Preliminary estimation of the basic reproduction number of novel coronavirus (2019-nCoV) in China, from 2019 to 2020: a data-driven analysis in the early phase of the outbreak. Int $J$ Infect Dis 2020;92:214-217.

5. Qiu Y, Wang S, Wang X, et al. Epidemiological analysis on a family cluster of COVID-19 [in Chinese]. Zhonghua Liu Xing Bing Xue Za Zhi 2020;41:506-509.

6. Yu P, Zhu J, Zhang Z, et al. A familial cluster of infection associated with the 2019 novel coronavirus indicating possible person-to-person transmission during the incubation period. J Infect Dis $2020 \mathrm{Feb} 18$ [Epub ahead of print]. doi: 10.1093/infdis/jiaa077.

7. Bae JM. A Chinese case of COVID-19 did not show infectivity during the incubation period: based on an epidemiological survey. J Prev Med Public Health 2020;53:67-69.

8. Lauer SA, Grantz KH, Bi Q, et al. The incubation period of coronavirus disease 2019 (COVID-19) from publicly reported confirmed cases: estimation and application. Ann Intern Med 2020;172:577-582.

9. Li Q, Guan X, Wu P, et al. Early transmission dynamics in Wuhan, China, of novel coronavirus-infected pneumonia. N Engl J Med 2020;382:1199-1207.

10. Tang $\mathrm{X}, \mathrm{Wu} \mathrm{C}, \mathrm{Li} \mathrm{X}$, et al. On the origin and continuing evolution of SARS-CoV-2. Natl Sci Rev 2020 [Epub ahead of print]. doi: 10.1093/nsr/ nwaa036. 